\title{
High efficiency DNA delivery into swine oocytes and embryos by electronic pulse delivery (EPD) ${ }^{1}$
}

\author{
YANG YANG ${ }^{2}$, SHAO HUA HUANG, XI ZHAO ${ }^{3}$ \\ InCell, Inc. 2310 Walsh Avenue, Santa Clara, California \\ 95051, USA.
}

\begin{abstract}
The production of transgenic swine for xenotransplantation has been proposed as an optimal option to overcome the chronic shortage of human organ donors. Generation of genetically engineered swine has been elusive due to the difficulties in gene transfer. In order to achieve effective gene delivery, a key step for the genetic modification, we applied electronic pulse delivery (EPD) technology to introduce $\mathrm{H} 2 \mathrm{~K}^{b}$-DC DNA construct into swine eggs. Using the developed EPD Protocols ${ }^{T M}$, we have achieved good viability of the EPD treated oocytes, satisfactory embryonic development of the EPD treated embryos, and stable DNA transfer into the swine embryos with high efficiency. Thus, application of the EPD technology promises to effectively facilitate the generation of large trangenic mammals.
\end{abstract}

Key words: Xenotransplantation, transgenic pig, electronic pulse delivery (EPD), swine embryos, DNA transfer.

The treatment of end-stage organ failure by allografting has been one of the

1. This publication is dedicated to Professor Lu Ji SHI's (L.C. Sze) 80th birthday

2. Present address: Department of Medicine, Division of Immunology and Rheumatology, Stanford University, School of Medicine, Stanford, CA 94305, Phone: (415) 723-7949 E-mail: yyang@leland, stanford. edu

3. Correspondence: Dr. Xi ZHAO, InCell, Inc. 2310 Walsh Ave. Santa Clara, CA 95051 Phone: (408) 492-9199; Fax: (408) 492-9101; E-mail: wxizhao@aol.com 
significant medical successes of the last two decades. Transplantation has been concentrated on allografting. The shortage of human donor organs in recent years has become a major obstacle to the fast growing transplantation therapy, and has stimulated huge efforts in xenotransplantation studies, especially the use of the organs from animals genetically modified to preclude immune rejection by the recipients. Today, 90,000 to 100,000 individuals could benefit from xenotransplantation, if such organ donors were available (UNOS Data).

Swine are considered as the most likely source of organs for xenotransplantation not only because of anatomical and physiological similarities of its organs to humans[1, 2], but also because the screening procedures have been carefully developed to prevent cross species transmission of viral or other types of infection from swine to humans[3, 4]. In addition, the large litter size of swine offers the advantage of the possibility of screening for a donor animal, and breeding such a donor animal to an adequate donor pool. However, swine organs engender substantial immunological hurdles. The first major hurdle is hyperacute rejection in primate recipients which destroys the graft within minutes to a few hours. It has been shown that hyperacute rejection is due to the human natural antibodies against Gal[ a 1-3] Gal expressed on the surface of swine endothelial cells and the activation of complement reactions[5]. One strategy to prevent hyperacute rejection in the porcine-to-primate xenografring model is to produce transgenic swine expressing high levels of human regulators of complement activation (RCAs), such as hCD 59 and decay-accelerating factor (DAF). Expression of RCAs by swine organs can block the formation of membrane attacking complex (MAC) and prevent the donor organ from complement mediated destruction[1, 6-8].

Great progress has been made to genetically modify animals since the first success in the early 1980 's. Microinjection is the most commonly used approach to introducing foreign DNA into recipient eggs to create transgenic animals. In small laboratory animals, such as mice and rats, the transgenesis rate is about $15-20 \%$ by microinjection. However, production of transgenic large farm animals by microinjection yields a very poor transgenesis rate ranging from $1 \%$ to $2 \%[9,10]$. One of the major reasons is that the microinjection technology is complicated by the invisible pronuclei of the embryos. In order to visualize the nucleus, extra handling step such as centrifugation is required which can significantly decrease the viability of the embryos[10, 11]. Thus, the key step to improve the transgenesis in swine is to develop an effective system to achieve high efficiency of DNA delivery into swine embryos with a high survival rate.

Electronic Pulse Delivery (EPD) has demonstrated its success to effectively introduce exogenous gene constructs and protein into a wide variety of cells including somatic cells, germ cells as well as stem cells[12, 13]. EPD employs precisely controlled electronic pulses which are defined and characterized by eight instrumentation parameters. The defined electronic pulses can be optimally programmed depending on the type and number of target cells to achieve a high delivery efficiency. Unlike 
conventional electroporation which results in severe damage of the target cells, EPD requires no contact between electrode(s) and cell suspension and creates a very low current ensuring excellent cell viability. Furthermore, the EPD procedure normally takes tens of seconds which limits possible damage caused by the engineering process itself and/or by the operating environment.

Having experienced the advantages of EPD technology in producing transgenic mice and fish[14, 15], we endeavored to develop an EPD protocol for high efficiency DNA delivery to swine embryos. In this communication, a DNA construct containing human CD59 cDNA driven by a mouse MHC class I H2 $\mathrm{K}^{b}$ promoter was transferred by EPD to swine oocytes and fertilized eggs. The viability and development potential of the oocytes and fertilized eggs which had been subjected to EPD treatment were evaluated systematically. The data reveals that high percentages of EPD treated oocytes and fertilized eggs survived and developed normally. Also, dot blot and PCR analysis demonstrated the presence of the transgene in a high percentage of embryos that were treated by EPD. The results of this study may not only lead to the creation of genetically engineered swine for xenotransplantation donors, but also be applied more broadly to the effective production of transgenic large farm animals for other human therapeutic applications.

\section{MATERIALS AND METHODS}

All culture media were from Gibco/BRL (Grand Island, NY) and the fetal bovine serum was purchased from Hyclone (Logan, UT). DNase I was purchased from Sigma (St, Louis, MO). H2K ${ }^{\mathrm{b}}$ DC DNA construct was kindly provided by Alexion (New Haven, CT), in which a human decayaccelerating factor and CD59 antigen chimeric cDNA was under the control of mouse MHC class I $\mathrm{H}_{2} \mathrm{~K}^{\mathrm{b}}$ promoter. T4 Nucleotide kinase was obtained from Amershan. The restriction enzymes and Taq polymerase were obtained from Promega (Medisine, WI).

\section{Collection of porcine oocytes}

Porcine oocytes were collected from ovaries which were obtained from a Modesto (California) slaughterhouse. The ovaries were separated from the reproductive tract immediately after slaughter and transported to the laboratory in warm saline solution at $35{ }^{\circ} \mathrm{C}$. The ovaries were then quickly rinsed with $75 \%$ alcohol followed by serial washes with saline solution. The cumulus masses were extracted from the ovaries'in m-PBS and the oocytes were collected under a stereomicroscope. The oocytes were cultured in TCM-199 with the supplements of $10 \%$ fetal bovine serum and FSH/LH at $38{ }^{\circ} \mathrm{C}$. The maturation of the oocytes was monitored and evaluated by microscopy.

\section{Harvest of fertilized eggs}

Donor giltes were stimulated with 1,500 IU/gilt Pregnant Mares' Serum Gonodotropin (PMSG) on the 14th day of estrous. $72 \mathrm{~h}$ post PMSG injection, each donor was primed with 500 IU Human Chorionic Gonadotrophin (hCG). The donors were then mated with boars $24 \mathrm{~h}$ after hCG injection. Porcine embryos at the 1-cell and 2-cell embryonic stage were collected $28 \mathrm{~h}$ after mating.

\section{EPD gene delivery}

Both oocytes and fertilized swine eggs were selected from the culture under a stereomicroscope. The maturing oocytes or fertilized eggs were transferred to EPD Transfer Buffer ${ }^{T M}$ and mixed with DNA constructs in an average volume of $100 \mu 1$. According to EPD Application Protocol ${ }^{T M}$, 
50 to 100 eggs were mixed with the Transfer Buffer containing the linearized plasmid DNA at a final concentration of $0.1 \mu \mathrm{g} / \mu \mathrm{l}$. The mixture was transferred to a Reaction Container ${ }^{T M}$ which was placed into the EPD Reaction Chamber ${ }^{T M}$ for EPD treatment. Various EPD Application Protocols ${ }^{T M}$ were selected for the specific applications. The EPD process takes tens of seconds. Based on our experience, the effects of room temperature can be ignored during such a short period of exposure time. The EPD treatment was completed by following the step-by-step EPD system screen instructions.

\section{Viability study and development potential evaluation of EPD treated cells}

After EPD treatment, the cells were transferred to a culture dish for the viability evaluation by Trypan blue exclusion. Some of the treated and control oocytes were cultured overnight and stained with Trypan Blue on the following day to confirm the viability of the EPD treated oocytes by light microscopy. The fertilized eggs were treated by EPD and followed by in vitro culture. Both treated and untreated control eggs were cultured in modified Whittens Medium (mWM) for further development. The embryonic cleavage and development were monitored by light microscopy.

\section{DNA transfer efficiency assessment}

The $\mathrm{H}_{2} \mathrm{~K}^{b}$-DC construct was radioactively end-labeled with ${ }^{32} \mathrm{P}$-ATP. The labeled DNA sample was then purified through a push column (Stratagene), precipitated and resuspended in an appropriate EPD Transfer Buffer ${ }^{T M}$. The quantity and quality of the DNA sample was traced by gel electrophoresis.

The labeled DNA was mixed with swine oocytes that were in the selected EPD Transfer Buffer $^{T M}$. The granulosa cells surrounding the swine oocytes had been removed before EPD treatment. For a control, a group of oocytes were incubated with radioactively labeled ${ }^{32} \mathrm{P}$ DNA without EPD treatment. After exposing to the EPD treatment, the oocytes in both the treated and the control groups were washed intensively with wash media containing $80 \mathrm{IU} / \mathrm{ml}$ (or $500 \mu \mathrm{g} / \mathrm{ml}$ ) DNase I. The oocytes were then transferred into m-PBS drops containing $0.1 \%$ Proteinase $\mathrm{K}$ to remove zona. Following three rinses, one "naked" oocyte at a time was placed onto a grid of nitrocellulose filter. The filter then was exposed to an X-ray film at $-20{ }^{\circ} \mathrm{C}$ for 7 to $10 \mathrm{~d}$.

\section{PCR analysis}

Each PCR reaction was carried out in a $25 \mu \mathrm{l}$ reaction mixture with DC primers to detect transgene. The $\beta 2$ primers were used to detect $\beta 2$ microglobulin gene in the embryos. The sequences of the primers are: DC53: ACGCTGGATATAAAGTCCACGCAG; DC55: CTGCAGTCCTCCACCTCCTCCTCTGCATTCAGGTGGT- GGG; $\beta$ 2-5: CCGAAGG-TTCAGGTTTACTCACGC; $\beta 2$-3: GGAGTGAACTCAGTGTGGACCAG. Each cycle consisted of denaturation at $94{ }^{\circ} \mathrm{C}$ for 90 sec, annealing at $58{ }^{\circ} \mathrm{C}$ for $2 \mathrm{~min}$ and polymerization at $72{ }^{\circ} \mathrm{C}$ for a total of 50 cycles. The expected PCR products are $822 \mathrm{bp}$ with DC primers and $200 \mathrm{bp}$ with $\beta-2$ primers. The amplified products were analyzed by electrophoresis on $2 \%$ agarose and visualized with ethidium bromide under UV lights.

\section{RESULTS}

\section{Viability of EPD treated oocytes and embryos}

The swine oocytes were used prior to the experiments using fertilized eggs in order to establish optimal culture conditions for swine embryos. After two days culture, $75 \%$ to $85 \%$ of the follicles in which the immature oocytes were surrounded by granulosa cells were extended. The extension of granulosa cells is believed to be an indicator of in vitro maturation of porcine oocytes. 
We next evaluated the effect of the EPD treatment on the viability of swine oocytes by Trypan blue exclusion. Both treated and untreated oocytes were either stained with Trypan blue immediately, or cultured for one day after being exposed to EPD. We have evaluated three selected EPD Application Protocols ${ }^{T M}$, and a total of 2,661 oocytes were analyzed. Based on the Trypan blue exclusion, $85 \%$ of the oocytes were alive in the control group while $71 \%$ to $79 \%$ of the EPD treated oocytes survived which represents $84 \%$ to $93 \%$ relative viability (Tab 1 ). The viability of EPD treated oocytes was slightly decreased when the oocytes were cultured for an additional one day. However, over $68 \%$ relative viability was obtained (Tab 2).

Tab 1. Viability of swine oocytes immediately after EPD treatment

\begin{tabular}{ccccc}
\hline EPD Protocols & $\begin{array}{c}\text { Total number } \\
\text { of oocytes }\end{array}$ & $\begin{array}{c}\text { Live } \\
\text { oocytes }\end{array}$ & $\begin{array}{c}\text { Viability } \\
(\%)\end{array}$ & $\begin{array}{c}\text { Relative viability of } \\
\text { EPD treated oocytes }\end{array}$ \\
\hline Control & 120 & 102 & 85.0 & $100.0 \%$ \\
Pro. 1 & 132 & 104 & 78.8 & $92.7 \%$ \\
Pro. 2 & 134 & 96 & 71.6 & $84.3 \%$ \\
Pro. 3 & 127 & 91 & 71.7 & $84.4 \%$ \\
\hline
\end{tabular}

The data are from five individual experiments. The viability of oocytes was determined by Trypan blue exclusion. The viability of oocytes was examined immediately after EPD treatment.

Tab 2. Viability of swine oocytes one day after EPD treatment

\begin{tabular}{ccccc}
\hline EPD Protocolsof & $\begin{array}{c}\text { Total number } \\
\text { oocytes }\end{array}$ & $\begin{array}{c}\text { Live } \\
\text { oocytes }\end{array}$ & $\begin{array}{c}\text { Viability } \\
(\%)\end{array}$ & $\begin{array}{c}\text { Relative viability of } \\
\text { EPD treated oocytes }\end{array}$ \\
\hline Control & 230 & 191 & 83.0 & $100.0 \%$ \\
Pro. 1 & 258 & 166 & 64.3 & $77.5 \%$ \\
Pro. 2 & 283 & 157 & 55.5 & $66.9 \%$ \\
Pro. 3 & 241 & 136 & 56.4 & $68.0 \%$ \\
\hline
\end{tabular}

The data are from five individual experiments. The viability of oocytes was determined by Trypan blue exclusion. The viability of oocytes was examined $24 \mathrm{~h}$ after EPD treatment.

The study was extended to the fertilized swine eggs with the selected EPD Application Protocols ${ }^{T M}$. We have adopted all three Protocols generated from oocyte studies plus two additional Protocols ${ }^{\mathrm{TM}}$ from our data base for the study of swine embryos. The embryos were cultured for $5 \mathrm{~d}$ after EPD treatment and the viability and development potential of these embryos were examined. We observed that over $50 \%$ of the 1-cell stage embryos were unable to develop further indicating the failure of fertilization in vivo. Therefore, we selected the 2-cell stage embryos in our study to ensure the comparable results between the EPD treated and the control embryos since the 2-cell stage embryos were fertilized and could develop further. As shown in Tab 3, over $60 \%$ of the embryos underwent normal development to the morula/blastula stage in a 5-day culture, indicating that the EPD Protocols ${ }^{T M}$ did not significantly hamper embryonic development. The embryos treated with 
Effective DNA delivery into swine embryos by EPD

Protocols ${ }^{T M} 4$ and 5 demonstrated better developmental ability compared to the embryos treated with Protocols ${ }^{T M} 1$ and 2 . There were no significant differences between the control and the EPD treated groups.

Tab 3. Development of swine embryos treated by EPD

\begin{tabular}{ccccc}
\hline EPD Protocols & $\begin{array}{c}\text { Total number of } \\
\text { embyros }\end{array}$ & $\begin{array}{c}\text { Enbryos to } \\
\text { morula stage }\end{array}$ & $\begin{array}{c}\text { Embryos to } \\
\text { blastula stage }\end{array}$ & $\begin{array}{c}\text { Morula and blastula } \\
\text { embryos }\end{array}$ \\
\hline Control & 57 & 28 & 17 & $78.9 \%$ \\
Pro. 1 & 137 & 17 & 7 & $64.9 \%$ \\
Pro. 2 & 42 & 17 & 9 & $61.9 \%$ \\
Pro. 3 & 59 & 39 & 12 & $86.4 \%$ \\
Pro. 4 & 46 & 24 & 21 & $97.8 \%$ \\
Pro. 5 & 36 & 24 & 9 & $91.7 \%$ \\
\hline
\end{tabular}

The 2-cell stage swine embryos were treated by selected EPD Protocols $^{T M}$ and then cultured for $5 \mathrm{~d}$. The number of embryos developed to the morula and blastula stages is summarized from 5 independent experiments. The data indicated that the EPD treatment did not significantly affect the viability and development potential of swine embryos.

\section{Analysis of EPD DNA transfer efficiency by Dot-blot and PCR}

Radioactive detection of Dot-blot and PCR analysis were employed to evaluate the EPD DNA transfer efficiency. The hDC construct was linearized and endlabeled. Following the purification, the labeled DNA was resuspended in Transfer Buffer $^{T M}$ for the EPD gene transfer. The control embryos were mixed with the radioactively labeled DNA with no EPD treatment. Both EPD treated and control embryos were then cultured for $2 \mathrm{~d}$ and then washed extensively with the medium containing DNase I to remove the DNA residual either from the surface of the embryos or the culture medium. In order to eliminate the non-specific signals, the zona was removed by proteinase K before the Dot-blot assay (Fig 1 A, B). Fig 2 shows the results from one of the typical experiments. The radioactive signals detected from the embryos indicated the presence of the transgene. It is noted that there was no signal in the control samples. The Dot-blot data from 3 independent experiments are summarized in Tab 4. No signal was detected in any of the control embryos among these three individual experiments, and the analysis of the embryos treated by the selected EPD Protocols ${ }^{T M}$ showed that the transfer efficiency ranged from $70 \%$ to $90 \%$.

Further studies were done to determine whether the transgene was stably present in the embryos during the embryonic development stages. The EPD treated embryos as well as the control groups were cultured in vitro for $5 \mathrm{~d}$ and the zona was removed by micro-manipulation under a dissecting scope. The embryos were then divided into 
Yany Y et al.

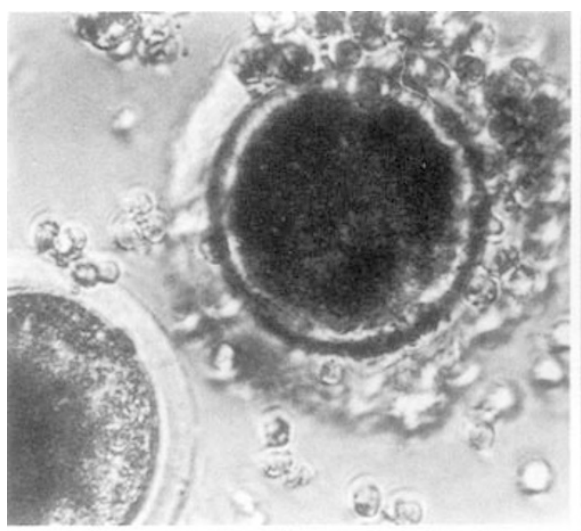

$\mathbf{A}$

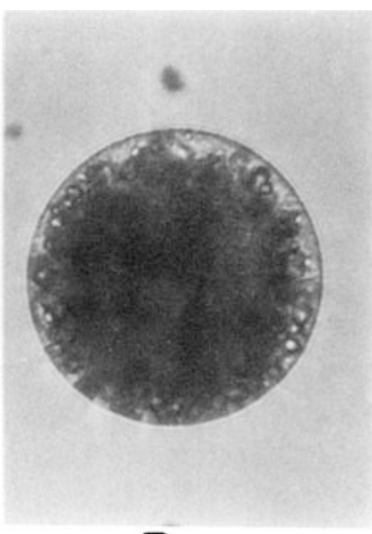

B

Fig 1. Swine eggs with zona surrounded by granulosa cells (A). After a brief proteinase $\mathrm{K}$ digestion, zona was removed, whereas the swine egg remained intact (B).

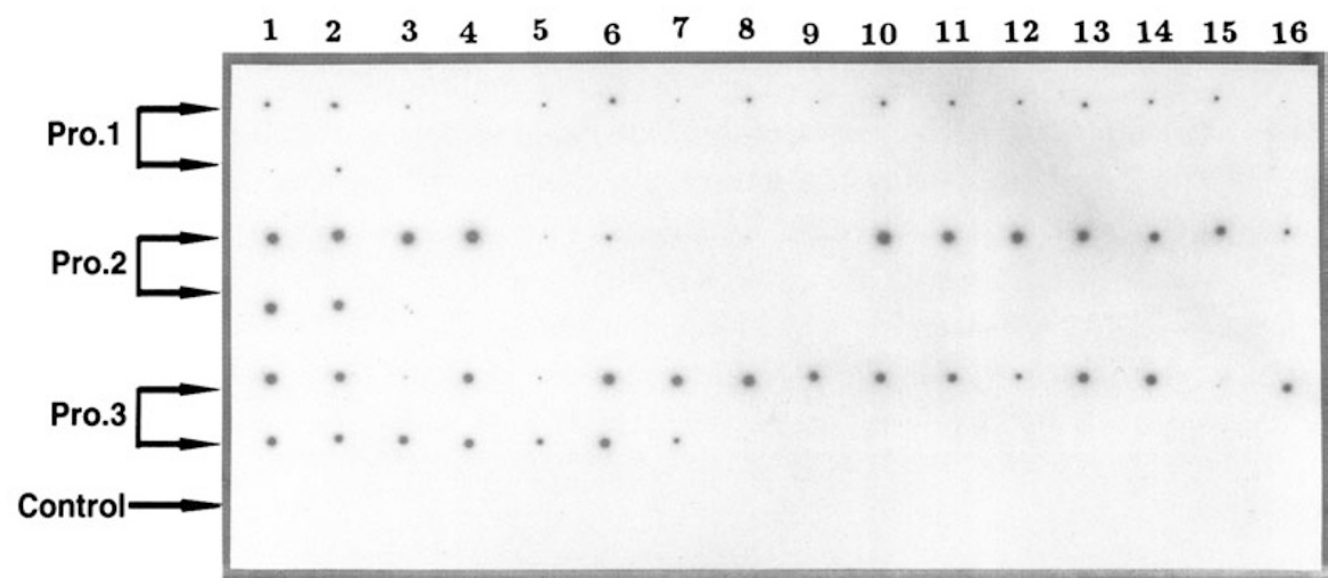

Fig 2. Autoradiography of a dot blot analysis for evaluation of DNA transfer efficiency of EPD Protocols ${ }^{T M}$. Swine eggs were mixed with ${ }^{32} \mathrm{P}$ end-labeled $\mathrm{hDC}$ construct (a $10 \mathrm{~kb}$ linearized fragment), and exposed to the selected EPD Protocols ${ }^{T M}$. After being cultured for two days, individual eggs were placed on nitrocellulose filter to detected the transgene positive eggs. The number of eggs in each group is as follows: Pro 1:18 eggs; Pro 2:19 eggs; Pro 3: 23 eggs and Control: 17 eggs. In the control group, there were no detectable radioactive signals though eggs were incubated with radioactive DNA without EPD treatment. Most of the eggs treated by EPD were transgene positive. The variation in radioactive signals may reflect various copy numbers of DNA transferred into the eggs. 
Effective DNA delivery into swine embryos by EPD

Tab 4. EPD DNA delivery efficiency evaluated by Dot-blot

\begin{tabular}{cccc}
\hline & & Dot blot positive vs. total & \\
EPD Protocols & Number of embryos & Dot blot positive & number of embryos $(\%)$ \\
\hline Control & 37 & 0 & 0 \\
Pro. 1 & 48 & 33 & $68.8 \%$ \\
Pro. 2 & 43 & 39 & $90.7 \%$ \\
Pro. 3 & 49 & 40 & $81.6 \%$ \\
Pro. 4 & 27 & 23 & $85.2 \%$ \\
Pro. 5 & 61 & 47 & $77.0 \%$ \\
\hline
\end{tabular}

DNA delivery efficiency was evaluated by dot blot analysis two d after EPD treatment. The zona was removed prior to the placement of individual embryos on the nitrocellulous filter to eliminate non-specific signals. There were no detectable signals in the control group. The five selected EPD Protocols ${ }^{T M}$ did not reveal significant differences.

two to four parts mechanically through micro-manipulation. Each part of the embryos was subjected to PCR analysis using DC primers for the detection of specific DC transgene, and $\beta-2$ primers for microglobulin gene. The amplified DC PCR products were detected in the EPD transferred embryos (Fig $3 \mathrm{~A}$ ), while only the $\beta$-2 band, not specific DC PCR products, was detected in the control group (Fig $3 \mathrm{~B})$. The PCR results for each EPD protocol from different experiments are summarized in Tab 5. As expected, the percentages of embryos showing the transgene positive was lower than those detected by Dot-blot indicating that some of the embryos lost the transgene during the embryonic cleavage, while some of the embryos possessed the transgene delivered by EPD.

$\begin{array}{lllllllll}1 & 2 & 3 & 4 & 5 & 6 & 78 & 9 & 10111213141551617181920212223242526\end{array}$

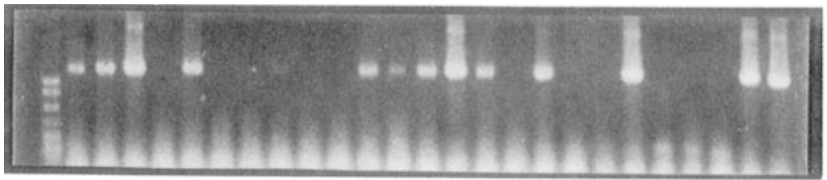

A $\begin{array}{llllll}1 & 2 & 3 & 4 & 5 & 6\end{array}$

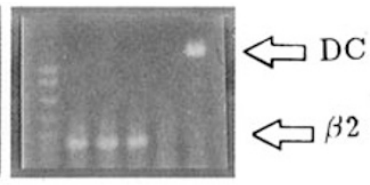

B

Fig 3. (A) Presence of DC transgene by genomic DNA PCR analysis. PCR products of DC transgene and swine $\beta 2$ microglobulin gene are indicated by arrowheads. The expected size of PCR products were $822 \mathrm{bp}$ and $200 \mathrm{bp}$, respectively. Lane 1 was molecular marker (pBR322/Msp I dig.); Lanes 2 to 26 are swine embryos transferred by EPD. (B) The result of DNA PCR analysis of one swine embryo in the control group. The embryo was cut into four sections and each section was subjected to a PCR detection with both pairs of primers of transgene and $\beta 2$ microglobulin gene. Lane 1: molecular marker; Lanes 2 to 5: sections of control swine embryos. In lane 6: a positive control for transgene PCR reaction. 
Tab 5. PCR analysis of the balstomeres of early stage embryos

\begin{tabular}{cccc}
\hline EPD protocols & $\begin{array}{c}\text { Total number of } \\
\text { embyros tested }\end{array}$ & $\begin{array}{c}\text { PCR positive } \\
\text { embryos }\end{array}$ & $\begin{array}{c}\text { PCR positive vs. total } \\
\text { number of embryos }(\%)\end{array}$ \\
\hline Control & 11 & 0 & 0 \\
Pro. 1 & 12 & 4 & $33.3 \%$ \\
Pro. 2 & 15 & 8 & $53.3 \%$ \\
Pro. 3 & 19 & 7 & $36.8 \%$ \\
Pro. 4 & 22 & 10 & $45.5 \%$ \\
Pro. 5 & 9 & 8 & $88.9 \%$ \\
\hline
\end{tabular}

The EPD treated and the control embryos were cultured for $5 \mathrm{~d}$. The embryos developed to morula/blastula stages were subjected to PCR detection for the transgene. Each individual embryo was dissected into 4 sections and followed by PCR reaction. There were no detectable signals in the control embryos.

\section{DISCUSSION}

The production of transgenic swine has been viewed as an optimal option to overcome the shortage of organ donors. There are several critical factors involved in the genetic modification of animals: the DNA consturct containing the transgene, the effective gene transfer process, the success of implantation and gestation, and the expression of the transgene. Efficient gene delivery, obviously, is the key step to create transgenic animals.

Since the EPD technology has previously demonstrated its advantages over the microinjection process, it was logical for us to apply it to achieve high efficiency of gene transfer for the production of transgenie swine. Therefore, we applied this novel gene transfer technology to deliver the hDC gene construct into swine eggs. We have demonstrated that the EPD treatment yielded good viability of the targets, both oocytes and fertilized eggs. More importantly, the EPD treated embryos developed normally. Over $65 \%$ of the EPD treated embryos developed to the morula/blastula stage. Though there were no data for a parallel comparison, the highest survival rate of the microinjected swine embryos was $12.8 \%[16,17]$. The low viability by microinjection may be due to mechanical damage, and/or the changes in intracellular microenvironment of the target eggs.

The EPD treated embryos were cultured for $2 \mathrm{~d}$ prior to the dot-blot analysis. The results demonstrated that $68.8 \%$ to $90.7 \%$ of the EPD treated embryos were transgene positive. The high percentage of presence of the transgene indicated a high DNA transfer efficiency by EPD. In order to follow the transgene during the early development, we performed PCR analysis of the divided embryos. This also enabled us to determine the homogeneity of the transgene bearing blastomeres. Our data showed that the gene construct delivered into the embryos at the 2-cell stage was stably present in the blastomeres at the morula and blastula stages, suggesting the integration of the transgene. The PCR results from the divided blastomeres also suggested even distribution of the transgene which were introduced into the targets at the 1-cell or 2-cell embryonic stage. 
In addition to the strategy of the blocking complement, the recent finding of the role of Gal[ $a$ l-3] Gal provides more detail for a road map towards the prevention of hyperacute rejection in porcine-to-primate xenotransplantation. Most of the pre-formed antibodies that are responsible for complement-mediated hyperacute rejection react with galactose in an a 1-3 linkage with galactose. Therefore, another strategy to the resistance to hyperacute rejection is to develop homologous recombination gene targeting in the porcine system to aim at completely eliminating the porcine Gal[ a l-3] Gal immunodominant by xenogenic engineering. Since this reported study opens the window for economical production of xenotransplantation donors, the EPD delivery technology may enable us to take advantage of its high DNA transfer efficiency to effectively create a reasonably sized pool for genetic selection.

The success of the effective DNA transfer into swine eggs also can be extended to the generation of other large animals which have previously demonstrated low transgenesis. For instance, genetically engineered goats and cows which are considered to be optimal bio-factories can be more efficiently generated by the EPD delivery process. EPD can also be applied to modify somatic cells that hold the potential for a therapeutic delivery system for gene therapy. The rejection-resistant donor animal tissues can be used as a therapeutic delivery vehicle. This application may be an alternative for the long term administration of certain therapeutics. It is obvious that EPD has a very board application spectrum in the pharmaceutical and therapeutically arena.

\section{REFERENCES}

[1] Cozzi E, White DJ. The generation of transgenic pigs as potential organ donors for humans. Nat Med 1995; 1 (9):964-6.

[2] Michaels MG, Simmons RL. Xenotransplant-associated zoonoses. Strategies for prevention. Transplantation 1994; 57 (1):1-7.

[3] Ye Y, Niekrasz M, Kosanke S, et al. The pig as a potential organ donor for man. A study of potentially transferable disease from donor pig to recipient man. Transplantation 1994; 57 (5) :694-703.

[4] Michaels MG, McMichael JP. Brasky K, et al. Screening donors for xenotransplantation. The potential for xenozoonoses. Transplantation 1994; 57 (10):1462-5.

[5] Sandrin MS, McKenzie IF. Gal alpha (1,3) Gal, the major xenoantigen(s) recognized in pigs by human natural antibodies. Immunol Rev 1994; 141:169-90.

[6] Dalmasso AP, Vercellotti GM, Platt JL, Bach FH. Inhibition of complement-mediated endothelial cell cytotoxicity by decay-accelerating factor. Potential for prevention of xenograft hyperacute rejection. Transplantation 1991; 52 (3):530-3.

[7] Fodor WL, Williams BL, Matis LA, et al. Expression of a functional human complement inhibitor in a transgenic pig as a model for the prevention of xenogeneic hyperacute organ rejection. Proc Natl Acad Sci USA 1994; 91 (23):11153-7.

[8] Ryan US. Complement inhibitory therapeutics and xenotransplantation. Nat Med 1995; 1 (9):967-8.

[9] Mullins J J, Mullins LJ. Transgenesis in nonmurine species. Hypertension 1993; 22 (4):630-3. 
[10] Janne J, Hyttinen JM, Peura T, et al. Transgenic bioreactors. Int J Biochem 1994; 26 (7):85970.

[11] Wall RJ, Pursel VG, Hammer RE, Brinster RL. Development of porcine ova that were centrifuged to permit visualization of pronuclei and nuclei. Biol Reprod 1985; 32 (3):645-51.

[12] Zhao X. EPD, a novel technology for drug delivery. Advanced Drug Delivery reviews 1995; 17: 257-62

[13] Zhao X. The Appylication of Electroic Pulse Delivery in Gene Therapy: progress and perspectives. In: Felgner PL, heller M J, Lehn P, Behr JP, Szoka FC. eds. Artificial Self-Assembling Systems for gene Delivery. The American Chemical Society 1996: 64-71.

[14] Zhao X, Zhang P J, Wong TK. Application of Baekonization: A new approach to produce transgenic fish. Molecular Marine Biology and Biotechnology 1993; 2: 63-69.

[15] Zhao X, Wong, TK. Production of transgenics by electronic pulse delivery. J Cell Biology 1991; 115:83.

[16] Langford GA, Yannoutsos N, Cozzi E, et al. Production of pigs transgenic for human decay accelerating factor. Transplant Proc 1994; 26 (3):1400-1.

[17] Rosengard AM, Cary NR, Langford GA, Tucker AW, Wallwork J, White DJ. Tissue expression of human complement inhibitor, decay-accelerating factor, in transgenic pigs. A potential approach for preventing xenograft rejection. Transplantation 1995; 59 (9):1325-33. 